\title{
XÂY DỰNG NĂNG LỰC ĐÁNH GIÁ CHO GIÁO SINH NGÀNH SƯ PHẠM TIẾNG ANH TẠI ĐẠI HỌC QUỐC GIA HÀ NỘI DỰA TRÊN NGUYÊN TÁC VỀ TÍNH GIÁ TRI
}

\author{
Dương Thu Mai*, Nguyễn Thị Chi, Phạm Thị Thu Hà \\ Khoa Su phạm tiếng Anh, Truờng Đại học Ngoại ngũu, ĐHQGHN, \\ Phạm Văn Đồng, Cầu Giấy, Hà Nội, Việt Nam \\ Nhận bài ngày 19 tháng 08 năm 2016 \\ Chỉnh sửa ngày 23 tháng 12 năm 2016; Chấp nhận đăng ngày 06 tháng 01 năm 2017
}

Tóm tắt: Trong bối cảnh năng lực đánh giá ngày càng trở nên quan trọng trong hệ thống các năng lực chuyên môn của giáo viên nói chung, và giáo viên dạy tiếng Anh nói riêng, nhóm nghiên cứu đã xây dựng một khung năng lực đánh giá tiếng Anh và bàn luận những vấn đề cần lưu ý khi xây dựng một khung năng lực như vậy về mặt phương pháp luận, cũng như về mặt cơ sở lý luận và thực tiễn. Cụ thể, các tác giả phân tích tính giá trị với vai trò là nguyên tắc cốt lõi của phương pháp xây dựng năng lực đánh giá, đồng thời trình bày quá trình áp dụng nguyên tắc này trong việc xây dựng năng lực đánh giá cho giáo sinh ngành Sư phạm tiếng Anh (SPTA) tại Trường Đại học Ngoại ngữ - Đại học Quốc gia Hà Nội (ĐHNN-ĐHQGHN). Thông qua phân tích tài liệu và phỏng vấn các chuyên gia về một khung năng lực đánh giá tiếng Anh ban đầu, dựa trên bảng câu hỏi về các khía cạnh của tính giá trị, các tác giả bàn luận về những cân nhắc thực tiễn và đối chiếu với những cân nhắc mang tính lý luận.

Tù khóa: năng lực đánh giá, tính giá trị, xây dựng khung năng lực

\section{Phần mở đầu}

Đánh giá (ĐG) năng lực tiếng Anh là một trong những nhiệm vụ thiết yếu đối với giáo viên tiếng Anh ở bậc phổ thông tại Việt Nam. Bộ tiêu chuẩn nghề nghiệp dành cho giáo viên tiếng Anh phổ thông do Bộ Giáo dục và Đào tạo (GD \& ĐT) ban hành năm 2013 gồm 5 lĩnh vực chuyên môn có quy định năng lực ĐG trong lĩnh vực 2. Cụ thể, tiêu chí này yêu cầu "giáo viên cần hiểu được các công cu và kỹ thuật ĐG trong ĐG thuờng xuyên và ĐG tổng kết, và có khả năng thiết kế và sủ dụng được các hình thức ĐG phù hợp với tù̀ng đối tượng học viên để cải tiến việc giảng dạy và đo luòng năng lục cũng nhu tiến bộ của học viên," (National Foreign Language 2020

\footnotetext{
* Tác giả liên hệ. ĐT.: 84-1669686968

Email: duongthumai@yahoo.com
}

Project, 2013). Ngoài ra, thông tư 30/2009/ TT-BGDĐT ban hành ngày 22 tháng 10 năm 2009 của Bộ GD \& ĐT quy định về chuẩn nghề nghiệp dành cho giáo viên trung học cơ sở, và trung học phổ thông cũng yêu cầu giáo viên có khả năng đánh giá kết quả học tập của học sinh, phát triển cho học sinh năng lực tự đánh giá và sử dụng kết quả đánh giá để cải tiến việc dạy và học (Trích thông tư 30/2009/ TT-BGDĐT). Bên cạnh đó, ĐG năng lực tiếng Anh còn là một nhiệm vụ trọng tâm trong Đề án quốc gia về đổi mới dạy và học ngoại ngữ giai đoạn 2008-2020 do Bộ GD \& ĐT chủ trì. Trong khuôn khổ đề án này, hàng nghìn giáo viên tiếng Anh công tác tại các cơ sở khảo thí được Bộ ủy quyền đã và đang tham gia ĐG năng lực tiếng Anh của đội ngũ giáo viên tiếng Anh các cấp, từ tiểu học đến đại học. 
Những thực tiễn trên cho thấy Bộ GD \& ĐT đã và đang thực hiện những thay đổi tích cực trong ĐG năng lực tiếng Anh. Bộ đã ban hành nhiều quy định và chính sách hướng dẫn cho kiểm tra ĐG ở các cấp học và ở các phạm vi khác nhau. Tuy nhiên, thực tế cho thấy vẫn còn tồn tại nhiều hạn chế trong quá trình nâng cao năng lực ĐG tiếng Anh cho giáo viên (Đặng, 2011) như hạn chế về nhân lực tham gia công tác ĐG, chưa có yêu cầu cụ thể, rõ ràng đối với các đối tượng tham gia ĐG, thiếu trình độ chuyên môn về ĐG, chưa có định nghĩa thành phần cụ thể của các kiến thức, kỹ năng ĐG. Các chính sách về chuẩn nghề nghiệp do Bộ GD \& ĐT ban hành cũng chỉ nêu yêu cầu chung chung về nhiệm vụ kiểm tra ĐG, do đó giáo viên còn lúng túng, khó khăn khi thực hiện nhiệm vụ công việc, các nhà đào tạo cũng gặp khó khăn khi xây dựng chương trình, khóa học về ĐG vì không có một nội hàm năng lực cụ thể để định hướng cho quá trình đào tạo. Có thể nói, các khung năng lực ĐG trong các chương trình đào tạo và bồi dưỡng giáo viên cũng như các quy định cho giáo viên về nhiệm vụ ĐG còn thiếu tính rõ ràng, tính đầy đủ và tính thuyết phục.

Xuất phát từ các điều kiện và vấn đề tồn tại từ thực tiễn nói trên, từ nhu cầu nhiệm vụ công việc của giáo viên giảng dạy tiếng Anh, nhóm nghiên cứu nhận thấy cần xây dựng một khung miêu tả nội hàm các thành phần của năng lực ĐG tiếng Anh theo một phương pháp tường minh, chú trọng tính khoa học và tính giá trị của khung. Trong bài báo này, các tác giả sẽ đưa ra những vấn đề mà người xây dựng năng lực nói chung và người xây dựng năng lực ĐG tiếng Anh nói riêng cần cân nhắc trong quá trình xây dựng và xác trị một khung năng lực như vậy, bắt đầu bằng các vấn đề phương pháp luận cho việc xây dựng năng lực nói chung, và sau đó là các vấn đề cụ thể về lý luận và phương pháp cho việc xây dựng năng lực ĐG tiếng Anh trong nghiên cứu này.

\section{Phương pháp xây dựng và xác trị một năng lực}

2.1. Nguyên tắc xây dựng năng lục - Khái niệm tính giá trị thống nhất

Trong các nghiên cứu giáo dục hiện đại, một khái niệm có thể đo lường và $Đ G$ được trong một bối cảnh cụ thể, ví dụ như một năng lực cụ thể, thường được gọi tên là một cấu trúc (construct), vì khái niệm này thường vẫn được xây dựng dựa trên các cơ sở lý thuyết cần thiết nhưng đã được điều chỉnh đi theo đặc điểm thực tế của một đối tượng nghiên cứu cụ thể. Vấn đề trọng tâm trong xây dựng một cấu trúc hoặc một năng lực là xác định được phương pháp và nguyên tắc thực hiện. Trong bài báo này, tính giá trị được nhóm tác giả lựa chọn là nguyên tắc quan trọng nhất, và từ đó trình bày một phương pháp xây dựng năng lực dựa trên nguyên tắc này.

Tính giá trị từng được định nghĩa đơn giản là sự phù hợp và chính xác của việc giải thích kết quả của một quá trình đo lường (Reynolds, Livingston, và Willson, 2006). Ba dạng giá trị thường được đề cập trước đây bao gồm: giá trị về nội dung (content), giá trị phân loại (criterion) và giá trị cấu trúc (construct). Từ những năm 70 , khái niệm tính giá trị có ý nghĩa bao quát hơn. Các nghiên cứu và thảo luận gần đây về tính giá trị đều chịu ảnh hưởng từ quan điểm của Samuel Messick, người đã liên tiếp định nghĩa và xây dựng khái niệm tính giá trị hợp nhất trong một chuỗi các nghiên cứu của mình $(1989,1990,1995)$, theo đó "tính giá trị là một nhận xét tổng hợp về mức độ những căn cứ khoa học và cơ sở lý thuyết có thể chứng minh sự đúng đắn 
và phù hợp của các nhận định ĐG về năng lực và của các hành động có liên quan tới kết quả ĐG”' (Messick, 1989, tr.5). Các loại giá trị không còn phân tách như trước mà đã tương quan với nhau và đều liên quan đến tính giá trị của cấu trúc/năng lực cần đo (Messick, 1990) (construct validity), bao gồm 6 khía cạnh (loại) như sau:

\begin{tabular}{|c|l|}
\hline Khía cạnh & \multicolumn{1}{|c|}{ Căn cứ xác định giá trị } \\
\hline $\begin{array}{c}\text { Nội dung } \\
\text { content) }\end{array}$ & $\begin{array}{l}\text { Sự liên quan và tính đại diện của nội } \\
\text { dung dùng đế đo lường so với khái } \\
\text { niệm cấu trúc đang được đo }\end{array}$ \\
\hline $\begin{array}{c}\text { Kết cấu } \\
\text { structural) }\end{array}$ & $\begin{array}{l}\text { Mối quan hệ tương quan của các } \\
\text { phần hoặc nội hàm: cấu trúc trong } \\
\text { Mối quan hệ tương quan giữa các } \\
\text { thang chấm và khung năng lực cần đo } \\
\text { Mối quan hệ tương quan của mức } \\
\text { năng lực đo được với các kết quả ĐG } \\
\text { khác hoặc với các đặc điểm khác của } \\
\text { người học: cấu trúc ngoài }\end{array}$ \\
\hline $\begin{array}{c}\text { Quá trình } \\
\text { (substantive) }\end{array}$ & $\begin{array}{l}\text { Sự hợp lý và đầy đủ của quá trình } \\
\text { thực hiện năng lực của người học }\end{array}$ \\
\hline $\begin{array}{c}\text { Khái quát hóa } \\
\text { (generalizability) }\end{array}$ & $\begin{array}{l}\text { Những điểm giống và khác nhau } \\
\text { trong quá trình thực hiện năng lực } \\
\text { trong những lần ĐG khác nhau } \\
\text { Độ dao động của kết quả sau khi } \\
\text { người học được hướng dẫn thêm }\end{array}$ \\
\hline $\begin{array}{c}\text { Ngoại vi } \\
\text { (external) }\end{array}$ & $\begin{array}{l}\text { Mối quan hệ giữa các điểm số ĐG } \\
\text { năng lực này và kết quả ĐG các năng } \\
\text { lực tương tự hoặc năng lực khác }\end{array}$ \\
\hline $\begin{array}{l}\text { Ý nghĩa sứ dụng của các nhận định } \\
\text { về điểm sồ, có xét tới các mục đích } \\
\text { ĐG ban đầu }\end{array}$ \\
\hline consequence)
\end{tabular}

Hình 1. Các khía cạnh của tính giá trị (Messick, 1990)

Chỉ khi các khía cạnh trên được quan tâm chứng minh thì một năng lực mới có đủ điều kiện để được công nhận là có giá trị. Càng nhiều khía cạnh được chứng minh thì tính giá trị càng được đảm bảo. Quan điểm của Messick về tính giá trị thống nhất, chú trọng cả nhận định về năng lực và cách sử dụng kết quả ĐG năng lực như trên, được nhiều chuyên gia giáo dục đầu ngành ủng hộ và tiếp tục phát triển (Popham, 2002; Reynolds và ctv, 2006). Đây có thể coi là một trong những lý thuyết về tính giá trị có ảnh hưởng lớn nhất hiện nay được ứng dụng trong ngành giáo dục, các dự án và nghiên cứu về ĐG giáo dục.

\subsection{Huớng tiếp cận xây dưng năng lực với} trọng tâm là tính giá trị

Trong việc xây dựng khung cấu trúc (construct framework) với các nội hàm, một mặt, người nghiên cứu có thể tiếp cận theo hướng định tính, dựa vào các dữ liệu thực tế và ý kiến chuyên gia. Mặt khác, quá trình xây dựng và xác trị một năng lực có thể thực hiện theo hướng tiếp cận định lượng. Điểm khác biệt của phương pháp tiếp cận theo hướng định lượng là nó chịu ảnh hưởng ít hơn từ ý kiến trực giác của các chuyên gia hoặc các phân tích thống kê trong việc phân loại độ khó của các tiêu chí ĐG. Tuy nhiên, dù là phát triển theo hướng tiếp cận định tính hay định lượng thì các khung năng lực hiện nay vẫn có một số nhược điểm nổi bật như: sự phụ thuộc vào trực giác của chuyên gia, dẫn tới thiếu cơ sở khoa học cho sự tồn tại của năng lực, sự mập mờ trong việc mô tả các cấp độ năng lực: "tốt", "rất tốt", sự thiếu liên quan tới nhóm đối tượng sử dụng khung năng lực, hoặc ngôn ngữ miêu tả không phù hợp và được vay mượn từ các khung năng lực cho đối tượng khác, sự đơn giản hóa quá mức quá trình thực hiện năng lực, dẫn tới miêu tả thiếu các năng lực thành phần.v.v. (Brindley, 1998; North và Schneider, 1998; Weigle, 2002).

Đáp lại những chỉ trích đó, Griffin, Adams, Martins và Tomlinson (1988) đã giới thiệu một phương pháp xây dựng khung năng lực có thể hạn chế được những hạn chế nói trên, và phù hợp với định nghĩa tính giá trị hợp nhất của Messick (1989). Dựa trên quy trình này, Griffin, Nguyen và Gillis (2004) đã đưa ra một quy trình chi tiết có thể áp dụng 
để đánh giá năng lực ngôn ngữ. Các bước thực hiện thiết kế năng lực, các minh chứng được thu thập trong từng bước thiết kế năng lực cũng như cách ĐG tính giá trị của năng lực qua từng bước sẽ được miêu tả trong mối liên hệ tới lý thuyết về tính giá trị thống nhất (Messick, 1989, 1990, 1995) cụ thể ở phần dưới đây.

\section{Bước 1: Phác thảo}

Ở bước này, nhà nghiên cứu đề xuất ra khung năng lực cùng các thành phần, xem xét lại các lý thuyết, nghiên cứu có liên quan và hoàn cảnh trong các cơ sở đó, từ đó tự thiết kế hoặc mời các chuyên gia cùng cộng tác trong việc phác thảo khung cấu trúc năng lực. Tất cả mọi khía cạnh của tính giá trị đều phải được cân nhắc, từ nội dung, kết cấu...cho tới hệ quả.

\section{Bước 2: Tham khảo ý kiến chuyên gia}

Trong bước này, một nhóm chuyên gia cùng xem xét bản phác thảo khung năng lực. Họ có thể là những người sử dụng chính công cụ này, bao gồm các giáo viên có kinh nghiệm, nhà nghiên cứu, v.v. Trong bước này, cơ sở lý thuyết của khung năng lực được ĐG từ góc độ của người sử dụng. Những tiêu chí và giá trị mà người sử dụng coi trọng được thể hiện ở bước thảo luận này, vì thế tính giá trị cấu trúc của công cụ ĐG được quan tâm rõ rệt và gắn liền với các giá trị tại bối cảnh ĐG.

\section{Bước 3: Thư nghiệm khung năng lực}

Bước 3 có sự trợ giúp từ một nhóm các chuyên gia giàu kinh nghiệm. Họ sử dụng khung năng lực đã qua xét duyệt để ĐG các sản phẩm năng lực, bài tập mẫu với chất lượng khác nhau, sau đó họ nhận xét về cách khung năng lực được sử dụng.
Bước 4: Áp dụng khung năng lục

Trong bước này, người nghiên cứu áp dụng khung năng lực đã qua ĐG thử nghiệm vào thực tế với một số lượng mẫu nhất định. Những người tham gia áp dụng đều đã hiểu rõ năng lực này trước khi tham gia. Số liệu được thu thập, làm rõ và phân tích nhằm tìm ra các minh chứng định lượng về cấu trúc năng lực. Các phương pháp ĐG cổ điển và hệ thống mô hình đo lường của Rasch được áp dụng trong bước này.

\section{Bước 5: Hoàn thiện khung năng lục}

Trong bước hoàn thiện công cụ, người thiết kế sẽ thảo luận các kết quả tường minh từ bước 4 cùng với các minh chứng và kết quả từ 3 bước trước. Tất cả minh chứng đã có về tính giá trị của khung năng lực được tích hợp để chỉnh sửa và hoàn thiện sản phẩm cuối cùng của khung năng lực.

Từ năm bước trên, quy trình đã liên tục kiểm tra và cập nhật minh chứng về các khía cạnh tính giá trị; trong đó các bước 1 và 2 tập trung vào các minh chứng định tính hơn so với các bước sau. Hướng tiếp cận chung về xây dựng và xác trị năng lực như vậy có thể áp dụng để xây dựng các năng lực chuyên biệt như năng lực ĐG tiếng Anh. Bài báo này tập trung vào những vấn đề được hé lộ khi thực hiện hai bước đầu tiên của quá trình xây dựng năng lực ĐG cho giáo sinh SPTA, ĐHNNĐHQG Hà Nội, trong đó khái niệm năng lục được sư dụng để chỉ mức độ vận dụng kiến thức, kỹ năng và thái độ của một học sinh khi thực hiện các nhiệm vu học tập. Năng lực ĐG bao hàm việc vận dụng kiến thức về ĐG tiếng Anh, kỹ năng và thái độ trong thực hiện quy trình ĐG tiếng Anh. Xây dựng năng lực ĐG tiếng Anh nghĩa là tìm hiểu tất cả những nhiệm vụ ĐG tiếng Anh chính yếu và những 
kiến thức, kỹ năng và thái độ phẩm chất mà người công tác trong lĩnh vực ĐG tiếng Anh cần nắm vững khi thực hiện những nhiệm vụ đó ở phạm vi công việc của họ. Phần tiếp theo sẽ trình bày các vấn đề lý luận và thực tiễn trong việc nghiên cứu xây dựng khung năng lực này, với trọng tâm vào các khía cạnh tính giá trị của năng lực như đã trình bày.

\section{Phác thảo khung năng lực ĐG ngôn ngũ - các cơ sở lý luận và thực tiễn}

Tính giá trị nội dung, kết cấu và chất liệu của năng lực ĐG của giáo sinh ngành SPTA, tại ĐHNN-ĐHQGHN, hay còn gọi là các giáo viên tiếng Anh bậc phổ thông trong tương lai, trước hết được xây dựng dựa trên các lý thuyết trong lĩnh vực ĐG giáo dục và ĐG tiếng Anh. Ngoài ra, nhóm tác giả cũng phân tích các cơ sở thực tiễn, bao gồm các bộ chuẩn năng lực ĐG dành cho giáo viên, và nội dung chính các khóa học ĐG tại các trường ĐH sư phạm trên thế giới, các yêu cầu về năng lực ĐG dành cho giáo viên và giáo viên tiếng Anh tại Việt Nam. Phần lớn các cơ sở này đã được mô tả kỹ trong một báo cáo trước đây (Dương và Phạm, 2012). Phần báo cáo dưới đây sẽ trình bày tóm tắt những nền tảng chính nói trên.

\section{1. Đặc điểm ĐG giáo dục hiện đại}

Xây dựng năng lực đánh giá cho một đối tượng cụ thể không thể bỏ qua các đặc điểm ĐG giáo dục hiện đại. Theo Birenbaum (1996), đường hướng tiếp cận của ĐGGD hiện đại là theo hướng "số nhiều" (pruralisic) thay vì theo hướng thái cực, đi theo một quan điểm duy nhất. Ngoài ra, mô hình ĐG hiện đại ít nhất phải thể hiện một sự "chuyển biến tư tưởng" (Cooper, 1997, tr.13) theo hướng lấy người học làm trung tâm ĐG. Cụ thể, mục đích ĐG phải rõ ràng và phù hợp, các nguyên tắc ĐG cần được đảm bảo, ví dụ như tính xác thực và tính giá trị, năng lực được ĐG và bài tập ĐG phải phức hợp, việc thực hiện các phương pháp ĐG mất thời gian và công sức hơn so với ĐG truyền thống. Trong các đặc trưng của hình thái ĐGGD hiện đại trên, hai đặc điểm là tính giá trị và độ tin cậy được nhắc tới trong hầu hết các tài liệu lý thuyết về ĐG, có ý nghĩa bao trùm với tất cả các khâu của quá trình ĐG (Dương, 2016). Như đã trình bày, đây cũng chính là những nguyên tắc quan trọng nhất cho việc xây dựng nội hàm khung năng lực ĐG trong nghiên cứu này.

\subsection{Các nhiệm vu chính trong quy trình $Đ G$}

Trong giáo dục nói chung, quy trình ĐG chuẩn đã được xây dựng tương đối đầy đủ, với những khâu và các lựa chọn cụ thể của từng khâu mà dựa vào đó ta có thể miêu tả một quy trình, kế hoạch ĐG nói chung và ĐG tiếng Anh nói riêng. Sơ đồ sau đây trình bầy các nhiệm vụ chính trong quy trình ĐG và quy trình ĐG năng lực tiếng Anh. Người thiết kế quy trình ĐG cần nắm vững các lựa chọn trong các khâu này.

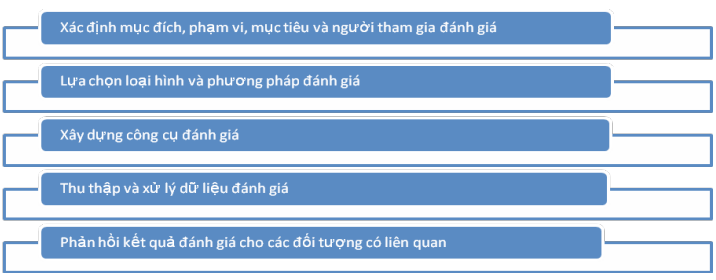

Hình 2. Quy trình ĐG (chỉnh sửa theo Dương và Phạm, 2012)

\subsection{Phân cấp năng lực theo mức độ tư duy}

Để xây dựng khung cho năng lực, ngoài vấn đề nội dung cần quan tâm tới mức độ thực hiện năng lực cho đối tượng của nghiên cứu. Trong phần này của báo cáo, nhóm nghiên cứu sẽ so sánh các mô hình tư duy trong giáo dục, từ đó tìm ra mô hình phù hợp nhất để phân cấp năng lực. Các học thuyết về phát 
triển tư duy trí tuệ hết sức đa dạng và phong phú, trong đó có mô hình của Bloom (1956) và Dreyfus và Dreyfus (1980). Bloom và các cộng tác viên (1956) đã xây dựng thang phân loại kiến thức theo mức độ tư duy bao gồm sáu bậc từ thấp đến cao: Biết, Hiểu, Vận dụng, Phân tích, Tổng hợp, Đánh Giá. Những năm 1990, thang tư duy của Bloom được chỉnh lại bởi Lorin Anderson, theo đó cấp bậc tư duy được đổi tên và chức năng (từ danh từ sang danh động từ) thành: Nhớ, Hiểu, Vận dụng, Phân tích, Đánh giá và Kiến tạo (Anderson và Sosniak, 1994). Ngoài ra, Dreyfus \& Dreyfus (1980) đã phát triển mô hình về quá trình một người học hình thành và phát triển kỹ năng của họ thông qua giảng dạy và thực hành. Quá trình này bao gồm năm mức phát triển, từ người tập sự đến chuyên gia: 1) Nguời tập sụ*; 2) Người bắt đầu, 3) Người có năng lục; 4) Nguò̀i thành thạo và 5) Chuyên gia.

So sánh các mô hình xây dựng khung năng lực theo hướng tư duy trên, mô hình của Dreyfus chủ yếu ĐG kỹ năng và kinh nghiệm, không ĐG kiến thức và vì thế không thật sự phù hợp cho việc xây dựng khung năng lực ĐG cho đối tượng giáo sinh SPTA của nghiên cứu mà phù hợp hơn với việc ĐG những người đã có nghề. Với thang phân loại tư duy của Bloom, bối cảnh giáo dục hiện nay đã thay đổi, mô hình tư duy của Bloom bị giới hạn trong việc đo lường các năng lực tư duy kiến thức hơn là năng lực thực hiện các kĩ năng, quá trình như ĐG tiếng Anh. Sau khi xem xét, so sánh đối chiếu các khung phát triển năng lực tư duy, trí tuệ, nhóm tác giả quyết định chọn mô hình phân cấp năng lực theo mức độ tư duy của Singer (2006) với định hướng tới một hệ thống phân cấp mức độ tư duy phù hợp với việc mô tả năng lực của người học với sáu cấp độ như sau:
1) Tiếp nhận (nhận diện các thuật ngữ, khái niệm, mối quan hệ, quá trình; quan sát hiện tượng, quá trình; nhận thức về các mối quan hệ, chuyển tiếp, quy trình; định nghĩa các khái niệm; thu thập dữ liệu từ các nguồn khác nhau);

2) Xử lý dữ liệu ở cấp độ 1 (so sánh các dữ liệu, thiết lập mối quan hệ; tính toán một phần kết quả; phân loại dữ liệu; biểu diễn dữ liệu; phân biệt; điều tra, khám phá; thử nghiệm);

3) Xử lý dữ liệu thông qua các mô hình, công thức (rút ra các mô hình; xác định các biến; thực hành theo các nguyên tắc/ mô hình chuẩn; giải quyết vấn đề thông qua các mô hình/thuật toán);

4) Mô tả, diễn đạt bằng ngôn ngữ của bản thân (miêu tả trạng thái, hệ thống, quy trình, hiện tượng; thiết lập và duy trì các lý lẽ để giải quyết vấn đề; trao đổi ý tưởng, khái niệm, giải pháp bằng ngôn ngữ hoặc tín hiệu riêng biệt cho lĩnh vực đó);

5) Xử lý cấp độ hai (so sánh các kết quả, sản phẩm, kết luận; tính toán kết quả; ĐG kết quả; phát triển học thuyết; phân tích các tình huống; xây dựng các chiến lược; liên kết các quá trình, tìm ra mối quan hệ giữa những đại diện, giữa những đại diện và các vật thể);

6) Chuyển giao (khái quát hóa và đặc thù hóa; tích hợp; tối đa hóa; chuyển hóa; thương thuyết; áp dụng các quy trình vào những tình huống phức tạp hơn).

Mô hình này tập trung vào kĩ năng xử lý kiến thức nhiều lần, qua nhiều giai đoạn, dựa trên quá trình. Đây cũng chính là quan điểm phổ biến về phát triển năng lực - trọng tâm của giáo dục hiện đại. Khung mô hình với 
6 giai đoạn cũng đặc biệt phù hợp với định nghĩa khái niệm "năng lực" bao hàm việc vận dụng kiến thức, kỹ năng, và thái độ. Mức độ 2 là mức độ yêu cầu người sử dụng năng lực nắm vững kiến thức, kỹ năng cơ bản và bước đầu áp dụng chúng trong thực hiện năng lực trong khi mức độ 3 phù hợp hơn với đối tượng đã thực sự vào nghề.

\section{Tiểu kết}

Sau khi nhóm tác giả nghiên cứu các cơ sở lý luận và thực tiễn về ĐG tiếng Anh, chúng tôi nhận thấy các năng lực thành phần sau luôn được nhấn mạnh trong hệ thống lý thuyết hiện đại về năng lực ĐG và ĐG tiếng Anh

- Thiết kế được một kế hoạch ĐG tiếng Anh với đầy đủ thành phần

- Thực hiện ĐG để phát triển học tập (nhấn mạnh mối quan hệ giữa ĐG với dạy, học tiếng Anh - Vai trò được chú trọng của ĐG trên lớp và đa dạng hóa phương thức ĐG trong giáo dục hiện đại)

- Thiết kế công cụ ĐG

- Xử lý số liệu trong ĐG bằng các mô hình đo lường và thống kê truyền thống cũng như hiện đại

- Phản hồi kết quả ĐG

Các năng lực này được mô tả với các đặc tả ở mức độ tư duy bậc 2 trên trong các cấp độ tư duy của Singer (2006). Nội dung của khung năng lực phác thảo làm rõ năm năng lực trên đây thành các nhiệm vụ nhỏ hơn với mức độ thực hiện rõ ràng, dựa trên các bước của quy trình đánh giá và các hành động thể hiện mức độ tư duy "xử lý dữ liệu thông tin ở cấp độ đơn giản". Ví dụ, năng lực thiết kế kế hoạch ĐGNN được làm rõ với nhiều nhiệm vụ đặc tả, trong đó có "hiểu các mô hình năng lực tiếng Anh", như các mô hình năng lực tiếng Anh giao tiếp. Một ví dụ khác, năng lực thực hiện $Đ G$ để thúc đẩy học tập và năng lực thiết kế công cụ đánh giá được làm rõ thành các nhiệm vụ sau (Bảng 1):

Bảng 1. Một số nhiệm vụ cụ thể trong năng lực ĐG và mức độ thực hiện

\begin{tabular}{|c|c|}
\hline $\begin{array}{l}\text { Thực hiện ĐG để } \\
\text { học tập: Kết hợp } \\
\text { đánh giá với quá } \\
\text { trình giảng dạy-học } \\
\text { tập tiếng Anh }\end{array}$ & $\begin{array}{l}\text { Nắm vững mối quan hệ biên } \\
\text { chứng giữa giảng dạy và đánh giá, } \\
\text { giữa kết quả học tập và việc thực } \\
\text { hiện ĐG kết quả ây; } \\
\text { Hiểu các nguyên tắc thiết kế kế } \\
\text { hoạch ĐG trên lớp; } \\
\text { Sử dụng các phương pháp ĐG trên } \\
\text { lớp lấy người học làm trung tâm; } \\
\text { Hiểu các yếu tố gây sai sót trong } \\
\text { quá trình đánh giá; } \\
\text { Nhận định về năng lực của HS dựa } \\
\text { trên kêt quả ĐG. }\end{array}$ \\
\hline $\begin{array}{c}\text { Thiết kế công cụ } \\
\text { đánh giá để phát } \\
\text { triển năng lực tiếng } \\
\text { Anh cho HS }\end{array}$ & $\begin{array}{l}\text { Phân biệt tầm quan trọng của các } \\
\text { loại công cụ ĐG khác nhau cho } \\
\text { mục đích ĐG trên lớp; } \\
\text { Sử dụng mô hình tư duy và năng } \\
\text { lực trong thiết kế mục tiêu ĐG; } \\
\text { Xây dựng một số thành phần bảng } \\
\text { thông số kỹ thuật của bài ĐG } \\
\text { Đánh giá về công cụ ĐG (tìm } \\
\text { điểm mạnh, điểm yếu); } \\
\text { Phát triển/Áp dụng công cụ ĐG } \\
\text { có sẵn để thúc đây việc học tập. }\end{array}$ \\
\hline
\end{tabular}

Khung năng lực cụ thể với từng nhiệm vụ và mức độ thực hiện của từng nhiệm vụ trên trong năng lực ĐG dành cho đối tượng giáo sinh ngành SPTA, ĐHNN-ĐHQGHN đã được trình bày cụ thể trong một báo cáo trước đây (Dương và Phạm, 2012). Trong bước phác thảo khung năng lực, nhóm nghiên cứu đã cân nhắc các khía cạnh của tính giá trị bao gồm tính đại điện và liên quan của các năng lực nội hàm với quá trình $Đ G$ tiếng Anh nói chung và của giáo viên tiếng Anh bậc phổ thông ở Việt Nam nói riêng, mối quan hệ giữa các năng lực nội hàm là hợp lý theo trình tự thời gian; các bước của quá trình ĐG đều được thể hiện đầy đủ, người thực hiện ĐG luôn cần thực hiện các năng lực này trong mỗi lần thực hiện ĐG, và mức độ tư duy của năng lực phù hợp với 
giáo sinh. Các khía cạnh khác của tính giá trị sẽ được làm rõ ở phần tiếp theo.

\section{Phỏng vấn chuyên gia về tính giá trị của khung năng lực ĐG}

Để khung năng lực ĐGNN cuối cùng có tính giá trị tốt hơn, nhóm nghiên cứu đã tiến hành tham khảo ý kiến các chuyên gia trong lĩnh vực SPTA và ĐG tiếng Anh. Cụ thể, 5 chuyên gia đã được mời tham gia với các thông tin trong bảng 2 :

Bảng 2. Thông tin các chuyên gia nhận xét

\begin{tabular}{|c|c|c|c|c|}
\hline $\begin{array}{c}\text { Chuyên } \\
\text { gia }\end{array}$ & Học vị & $\begin{array}{c}\text { Giới } \\
\text { tính }\end{array}$ & $\begin{array}{c}\text { Số năm } \\
\text { trong ngành }\end{array}$ & Chức vụ \\
\hline Q1 & Tiến sỹ & Nữ & 18 & $\begin{array}{c}\text { Giám Đốc } \\
\text { Trung Tâm } \\
\text { Nghiên Cứu }\end{array}$ \\
\hline H1 & Tiến sỹ & Nũ & 30 & $\begin{array}{c}\text { Chuyên gia } \\
\text { ĐG và giảng } \\
\text { viên môn } \\
\text { ĐG }\end{array}$ \\
\hline H2 & Tiến sỹ & Nữ & 30 & $\begin{array}{c}\text { Chuyên gia } \\
\text { ĐG, Trưởng } \\
\text { Khoa }\end{array}$ \\
\hline T1 & $\begin{array}{c}\text { Phó Giáo } \\
\text { Sư }\end{array}$ & Nam & 20 & $\begin{array}{c}\text { Trưởng } \\
\text { Khoa, } \\
\text { Chuyên gia } \\
\text { ĐG ngoại } \\
\text { ngữ }\end{array}$ \\
\hline H3 & Tiến sỹ & Nam & 25 & $\begin{array}{c}\text { Giám đốc } \\
\text { trung tâm } \\
\text { nghiên cứu, } \\
\text { Chuyên gia } \\
\text { ĐG }\end{array}$ \\
\hline
\end{tabular}

Nhóm nghiên cứu bắt đầu cuộc phỏng vấn bằng việc giải thích thuyết minh đề tài, giới thiệu về năng lực ĐG tiếng Anh và cách phác thảo năng lực ĐG tiếng Anh, trình bày về bản phác thảo khung năng lực cho các chuyên gia. Trong phần đầu của cuộc phỏng vấn, ý kiến của các chuyên gia về tính đại diện, tầm quan trọng, sự rõ ràng, tính logic của từng thành phần khung năng lực được ghi lại. Phần này mất tương đối nhiều thời gian nhưng là cần thiết để đảm bảo chuyên gia nắm được cả khung năng lực ĐG tiếng Anh. Ở phần sau, các chuyên gia được mời trả lời phỏng vấn bán cấu trúc theo các câu hỏi có trọng tâm là các khía cạnh tính giá trị sau:

a) Tính đầy đủ của nội dung khung năng lực

b) Tính đại diện của nội dung khung năng lực

c) Sự phù hợp của các tiêu chí trong từng năng lực thành phần với từng năng lực thành phần

d) Sự logic, phù hợp và cân đối trong kết cấu (structural aspect) khung năng lực

e) Độ rõ ràng (structural aspect) của kết cấu khung năng lực

f) Tính khả thi

\section{g) Tính khái quát hóa}

Các câu hỏi phỏng vấn được thiết kế dựa trên các khía cạnh khác nhau của tính giá trị để đảm bảo chuyên gia có thể cho ý kiến về các mặt giá trị khác nhau của khung năng lực. Người phỏng vấn cũng thường xuyên hỏi thêm các câu hỏi phụ để làm rõ hơn câu trả lời của chuyên gia. Kết quả các cuộc phỏng vấn được nhóm tác giả phân tích theo ý chính và chủ đề, và tập hợp lại dưới các khía cạnh như sau:

\section{Tính đặc thù/đại diện}

Tính đặc thù hay tính đại diện của nội dung năng lực là khía cạnh tính giá trị được các chuyên gia quan tâm nhiều nhất khi xem xét khung năng lực. Theo họ, việc xây dựng và áp dụng khung năng lực cần xem xét các nội hàm phù hợp, mang tính đại diện cho một đất nước hay một địa phương cụ thể. Các chuyên gia gợi ý để đảm bảo tính đặc thù hay đại diện của khung năng lực, người thiết kế cần cân nhắc các điều kiện về nhân lực, vật lực, cũng như 
các chính sách giáo dục trong quá trình xây khung năng lực. Các chuyên gia nhấn mạnh rằng một khung năng lực đảm bảo tính đặc thù có thể làm tăng tính khả thi trong việc áp dụng rộng rãi khung năng lực đó. Ở đây có sự giao thoa giữa khía cạnh nội dung và khía cạnh khái quát hóa của tính giá trị. Ví dụ, trong ĐG giáo dục có mô hình đo lường theo lý thuyết ĐG cổ điển và hiện đại. Tuy nhiên, nguồn đầu tư cho các công cụ// phần mềm dùng theo lý thuyết ĐG hiện đại còn hạn chế ở nhiều cơ sở đào tạo Việt Nam, trong khi đó các công cụ/ phần mềm theo lý thuyết ĐG cổ điển khá phổ biến, quen thuộc. Trong trường hợp này, nếu khung năng lực ĐG năng lực áp dụng mô hình đo lường theo cả lý thuyết cổ điển và hiện đại thì sẽ gây không ít khó khăn, trở ngại cho giáo sinh, giáo viên hướng dẫn môn học cũng như cho giáo viên nói chung. Như vậy, để đào tạo năng lực ĐG này trên diện rộng, việc lựa chọn các mô hình đo lường theo lý thuyết cổ điển được xem là hợp lý hơn, đã xét đến yếu tố đặc thù, mang tính địa phương. Tuy vậy, nếu giáo viên và nhà trường cụ thể có điều kiện thì vẫn có thể cân nhắc tích hợp giảng dạy cả các đặc điểm cơ bản của lý thuyết ĐG hiện đại. Trong việc cân nhắc giá trị nội dung của nội hàm năng lực và giá trị thực tiễn của việc sử dụng năng lực, dường như các chuyên gia cho rằng người thiết kế năng lực cần dựa trên đặc điểm thực tiễn hơn là khía cạnh nội dung. Nhóm nghiên cứu hoàn toàn đồng ý với quan điểm này của các chuyên gia.

\section{Tính khái quát}

Bên cạnh đó, một vấn đề thực tiễn khác được đặt ra là khung năng lực ĐG cần đại diện cho phổ làm việc của giáo viên. Theo các chuyên gia, giáo viên phổ thông hiện nay vẫn phải tiến hành đồng thời ĐG thường xuyên để phát triển học tập và ĐG tổng hợp thông qua các bài kiểm tra cuối kì, các bài thi sát hạch. Thậm chí, có giáo viên còn tham gia vào quá trình ĐG các bài thi học sinh giỏi của thành phố, của sở. Thực tế cũng cho thấy giáo viên phổ thông có thể được giao nhiệm vụ ra đề thi. Chưa nói đến việc họ có thể tự thiết kế một đề thi hoàn chỉnh thì ít nhất họ cũng cần $Đ G$ được câu hỏi nào có thể phù hợp cho mục đích ĐG tại một thời điểm cụ thể. Đây là những năng lực nội hàm vô cùng cần thiết và chỉ có thể hiệu quả nếu được đào tạo một cách bài bản. Ngoài ra, sau khi ra trường giáo sinh có thể trở thành giáo viên phổ thông hoặc ở các cấp đào tạo cao hơn; do vậy năng lực ĐG của giáo sinh cần thiết thực, gắn liền với các nhiệm vụ ĐG mà giáo viên phải thực hiện. Để đảm bảo tính khái quát của khung năng lực, người thiết kế cần hiểu quy định cũng như thực tế công việc của giáo viên ở diện rộng, ở nhiều cơ sở đào tạo khác nhau và các cấp học khác nhau. Theo ý kiến chuyên gia, phần lớn các tiêu chí nội hàm nhóm nghiên cứu lựa chọn cho khung năng lực ĐG đã bao hàm được các nhiệm vụ ĐG cơ bản này của giáo viên phổ thông và chuẩn bị cho họ khi bước vào nghề. Tuy nhiên, một số nhiệm vụ đặc biệt như thiết kế bài thi diện rộng và bài thi tổng kết thì chưa được chú trọng đủ. Về ý kiến này, quan điểm của nhóm nghiên cứu là cân nhắc và giới hạn lại một số năng lực nội hàm cơ bản nhất, do điều kiện thực tế khó có thể đào tạo hết cho giáo sinh về mọi nhiệm vụ ĐG mà tất cả các đối tượng giáo viên phổ thông có thể đảm nhiệm trong quá trình công tác của họ. Với những nhiệm vụ đặc biệt như thiết kế bài thi học sinh giỏi, thiết kế bài thi chuẩn hóa diện rộng, giáo viên khi ra trường vẫn cần tiếp tục được bồi dưỡng chuyên môn. Như vậy tính đại diện trong giá trị nội dung cần được đặt so sánh với tính khả thi trong đào tạo năng lực ĐG. 


\section{Độ rõ ràng}

Các chuyên gia đặc biệt chú ý đến văn phong trong mô tả các tiêu chí nội hàm của khung năng lực. Trên thực tế, lý thuyết về ĐG phần lớn được viết bằng tiếng nước ngoài, và việc mô tả khung năng lực bằng tiếng Việt là một thách thức không nhỏ.

Thứ nhất, văn phong của hai ngôn ngữ khác nhau, do đó việc chuyển ngũ̃ đòi hỏi người thiết kế cần cân nhắc rất kĩ lưỡng. Các cân nhắc này có tác dụng tăng khả năng hiểu và áp dụng rộng rãi khung năng lực, đặc biệt đối với giáo sinh hay giáo viên mới làm quen với khung năng lực ĐG.

Thứ hai, nét nghĩa rộng hẹp và sự quen thuộc của một số từ ngữ cũng là một vấn đề cần quan tâm. Khi nói "lựa chọn, xác định mức và nội dung chuẩn đầu ra ngoại ngữ phù hợp cho một đối tượng học sinh", người đọc có thể nhầm tưởng "chuẩn đầu ra" là một khái niệm rộng như chuẩn năng lực ngôn ngữ. Đây là một điều dễ hiểu vì từ trước đến giờ hầu hết giáo sinh, giáo viên đều đã quen thuộc với thuật ngữ "chuẩn đầu ra" và xem đó như một cách nói ngắn gọn đối với những chuẩn như khung tham chiếu châu Âu. Với những trường hợp như thế này, cách khắc phục tốt nhất là thay đổi từ ngữ cho phù hợp hơn với ngữ cảnh sử dụng.

Thứ ba, đối với một chuyên ngành như ĐG, việc sử dụng các thuật ngữ trong mô tả năng lực là điều tất yếu. Tuy nhiên, nhiều thuật ngữ chưa có từ tương đương trong tiếng Việt hoặc cách chuyển ngữ còn nhiều tranh cãi trong giới chuyên gia. "Phương pháp thay thế” là một ví dụ điển hình về sự không thống nhất giữa cách dịch và cách hiểu thuật ngữ. Cụm từ này gắn với thuật ngữ "alternative assessment" tức là các phương pháp ĐG khác với phương pháp ĐG truyền thống (bài thi), bao gồm: quan sát lớp, đặt câu hỏi, phỏng vấn, hay bài tập lớn. Xét về nghĩa, "alternative" dược dịch là "thay thế", và "assessment" được dịch là "ĐG”. Như vậy dịch "alternative assessment" là "phương pháp ĐG thay thế" là khá phù hợp. Tuy nhiên, với một người không có chuyên môn về kiểm tra ĐG có thể sẽ hiểu cụm từ "phương pháp thay thế" theo hướng: phương pháp khác so với phương pháp đã được dùng; ví dụ trước đây dùng phương pháp “đặt câu hỏi”, bây giờ không dùng phương pháp đó nữa mà thay thế bằng phương pháp "giao bài tập lớn”. Do đó, có chuyên gia đề xuất cách dịch 'phương pháp ĐG phi truyền thống,' thay vì sử dụng 'phương pháp ĐG thay thế.' Bên cạnh thuật ngữ "alternative assessment" còn nhiều thuật ngữ khác gặp vấn đề tương tự như "test specification" (tạm dịch là "bảng đặc tả kĩ thuật"), "matrix" (ma trận), hay "validation" (tạm dịch là "thẩm định"). Có chuyên gia cho rằng "thẩm định" trong tiếng Việt không chỉ mô tả một hoạt động "kiểm tra lại sản phẩm" mà còn nói đến đối tượng kiểm tra là người khác với người tạo ra sản phẩm đó. Tuy nhiên, cách hiểu này chưa được chính thức công nhận. Vấn đề dịch các khái niệm từ tiếng Anh sang tiếng Việt thực sự là một trở ngại vì một khung năng lực được mô tả thuần Việt có thể gây tranh cãi hoặc hiểu theo các cách khác nhau. Một trong các cách khắc phục mà nhóm nghiên cứu áp dụng cho vấn đề này là vừa dùng chuyển ngữ vừa dùng thuật ngữ gốc khi mô tả các tiêu chí nội hàm.

\section{Tính khả thi}

Các chuyên gia đề cập đến việc cân nhắc thời lượng đào tạo về năng lực cũng như độ sâu rộng của kiến thức được cung cấp khi xét tính khả thi của khung năng lực. Ví dụ, đối với tổng hợp kết quả định lượng, để thông 
hiểu các thuật toán trong xác xuất thống kê đòi hỏi khả năng tư duy và thời gian tiếp nhận phù hợp. Với khuôn khổ một khóa học diễn ra trong một kì học, người học khó có thể xử lý các thông tin phức tạp trong lý thuyết cũng như thực hành. Vì vậy, với giáo sinh có thể chỉ cần hiểu ý nghĩa của giá trị trung bình, độ lệch chuẩn, phân phối chuẩn là phù hợp. Một ví dụ khác liên quan đến sản phẩm mục tiêu của khung năng lực là việc xây dựng bảng thông số kĩ thuật (test specification). Đây được xem là một nhiệm vụ khó khăn, và để ra được sản phầm là một bảng thông số kĩ thuật đơn giản cũng mất khá nhiều thời gian. Hơn nữa, các tiêu chí nội hàm phải được lựa chọn phù hợp với khả năng tiếp nhận của người học. Với khả năng tri nhận của giáo sinh năm thứ tư, theo phân tích của chuyên gia, "phân biệt" giống như "khu biệt", tức là phải nắm được nhiều vấn đề liên quan, nên khi tích hợp tiêu chí "phân biệt" các mô hình tư duy hay các khung tham chiếu trong ĐG cần cân nhắc khả năng tri nhận của giáo sinh. Với một khung lý thuyết ở tầm vĩ mô như mô hình tư duy hay khung tham chiếu, đối với giáo sinh chỉ nên dừng ở mức "nhận biết". Việc xây dựng năng lực ĐG cần cân nhắc kỹ hơn tới yêu cầu về đến tính khả thi này.

Kết quả phỏng vấn như trên đã làm rõ thêm các vấn đề cần được quan tâm trong việc xây dựng và điều chỉnh năng lực ĐG trong hoàn cảnh giáo dục cụ thể cho giáo sinh ngành SPTA, ĐHNN-ĐHQGHN. Phần lớn các vấn đề chuyên gia nêu ra là các vấn đề thực tiễn và từ đó làm rõ một khía cạnh giá trị nào đó của năng lực ĐG được đề xuất.

\section{Kết luận}

Báo cáo này đã làm rõ một số khía cạnh tính giá trị cần được người thiết kế năng lực chú ý. Trong bước phác thảo, họ có thể quan tâm tới các khía cạnh sau: 1) giá trị nội dung; 2) giá trị kết cấu; 3) giá trị quy trình; 4) giá trị thực tiễn/hệ quả. Trong bước lấy ý kiến chuyên gia để bước đầu xác trị năng lực, các khía cạnh giá trị về độ khả thi và khái quát và giá trị ngoại vi thường được các chuyên gia cho ý kiến xác đáng hơn, do chuyên môn và tầm hiểu biết thực tế của các chuyên gia thường rộng hơn của nhóm nghiên cứu. Tuy nhiên, các ý kiến chuyên gia cũng cần được cân nhắc bên cạnh các khía cạnh khác của tính giá trị đã cân nhắc từ bước phác thảo, để từ đó đưa ra một cấu trúc năng lực vừa đảm bảo tính lý luận vừa đảm bảo tính thực tiễn. Cụ thể, nhóm nghiên cứu đã thực hiện các chỉnh sửa sau với khung năng lực ĐG dành cho giáo sinh sau khi nhận được ý kiến của các chuyên gia:

- Giảm các nhiệm vụ liên quan tới lý thuyết ĐG hiện đại trong năng lực sử dụng các lý thuyết ĐG trong xử lý dữ liệu ĐG, chỉ tập trung hướng giáo sinh tới việc hiểu và áp dụng một số nội dung trong lý thuyết ĐG cổ điển.

- Giữ nguyên các nhiệm vụ ĐG cơ bản như đã phác thảo, do khó có thể đào tạo thêm những nhiệm vụ chuyên biệt khác của giáo viên (như là thiết kế đề thi học sinh giỏi) với giới hạn của chương trình đào tạo giáo sinh.

- Sử dụng thuật ngữ với cả phiên bản tiếng Anh và tiếng Việt để tránh hiểu nhầm

- Giảm bớt yêu cầu trong một số nhiệm vụ, không yêu cầu giáo sinh phải thực hiện nhiệm vụ ở mức phức tạp, mà chỉ cần nhận biết và hiểu về nhiệm vụ. Ví dụ, nhiệm vụ xây dựng một số thành phần của bảng đặc tả kỹ thuật bài ĐG (trong 
năng lực thiết kế công cụ ĐG) tương đối phức tạp nên không được giữ lại. Cụ thể, năng lực Thiết kế công $c u$ ĐG để phát triển năng lục ngoại ngũ được điều chỉnh và chỉ bao hàm các nhiệm vụ: Phân biệt tầm quan trọng của các loại công cụ ĐG khác nhau cho mục đích ĐG trên lớp; Sư dưng các loại mô hình tư duy và năng lực trong thiết kế mục tiêu ĐG; Nhận xét về chất luợng công cụ ĐG; Phát triển/Áp dụng công cụ ĐG để thúc đẩy học tập.

Khung năng lực sau khi chỉnh sửa nhìn chung vẫn có cấu trúc như khung đã phác thảo, chỉ điều chỉnh về số lượng các nhiệm vụ trong năng lực, và mức độ thực hiện các năng lực. Một kinh nghiệm quan trọng nhóm nghiên cứu có thể kết luận là lấy ý kiến chuyên gia chưa phải là bước cuối cùng của quá trình xác trị năng lực. Tính giá trị là một khái niệm tương đối và khó có thể hoàn toàn đạt được mức độ tối cao. Các bước tiếp theo trong xây dựng khung năng lực ĐG sẽ được thực hiện và quá trình xác trị sẽ tiếp tục tìm ra các vấn đề khác mà người xây dựng năng lực cần quan tâm.

\section{Tài liệu tham khảo}

\section{Tiếng Việt}

Dương Thu Mai (2016). Hình thái ĐG hiện đại và các phương pháp ĐG năng lực học tập của học sinh. Tạp chí Khoa học Đại Học Quốc Gia Hà Nội, 32(1), 51-61, Hanoi.

Đặng Thị Thanh Huyền (2011). Khảo sát thực trạng đào tạo, bồi dưỡng về kiểm tra ĐGGD ở Việt Nam. Hanoi: Vietnam Ministry of Education and Training. Báo cáo chuyên gia chương trình READ Việt Nam.

Thông tư 30/2009/TT-BGDĐT quy định chuẩn nghề nghiệp giáo viên trung học cơ sở, giáo viên trung học phổ thông.

\section{Tiếng Anh}

Anderson, L. W., \& Sosniak, L. A. (Eds.). (1994). Bloom's taxonomy: A forty-year retrospective. Ninety-third year book of the National Society for the Study of Education. Chicago: University of Chicago Press.

Birenbaum, M. (1996). Assessment 2000: Towards a pluralistic approach to assessment. In M. Birenbaum \& F. Dochy (Eds.), Alternatives to assessment of learning achievements, process, and prior knowledge (pp. 3-29). Boston, MA: Kluwer Academic.

Bloom, B., Englehart, M. Furst, E., Hill, W., \& Krathwohl, D. (1956). Taxonomy of educational objectives: The classification of educational goals. Handbook I: Cognitive domain. New York, Toronto: Longmans, Green.

Brindley, G. (1998). Describing language development? Rating scales and second language acquisition. In L. F. Bachman \& A. D. Cohen (Eds.), Interfaces between second language acquisition and language testing research. Cambridge: Cambridge University Press.

Cooper, C. h. (1997). Learner-centred assessment. Launceston, Tasmania: Global Learning.

Dreyfus, S.E. \& Dreyfus, H.L. (1980). A fivestage model of mental activities involved in directed skill acquisition. Research report.

Dương, T. M. \& Phạm, T. T. H (2012). Building an assessment competence framework for pre-service and in-service TEFL teachers at Vietnam National University. Paper presented and printed in the Proceedings of SEAMEO RETRACT TESOL Conference, Danang, Vietnam.

Griffin, P., Nguyen, T. K. C., \& Gillis, S. (2004). An empirical analysis of primary teacher standards in Vietnam: Curricular reforms in Southeast Asia, planning and changing. Paper presented at the ICET World Assembly 2004. 
Messick, S. (1989). Meaning and Values in Test Validation: The Science and Ethics of Assessment. Educational Researcher, 18(2), 5-11.

Messick, S. (1990). Validity of Test Interpretation and Use. Princton, New Jersey: Educational Testing Service.

Messick, S. (1995). Validity of Psychological Assessment: Validation of Inferences from Persons' Responses and Performances as Scientific Inquiry into Score Meaning. American Psychologist, 50(9), 741-749.

National Foreign Language 2020 Project, Vietnam National Institute for Educational Sciences, Ministry of Education and Training (2013). Competency framework for English language teachers: user's guide. Hanoi: Vietnam Education Publishing House.
North, B., \& Schneider, G. (1998). Scaling descriptors for language proficiency scales. Language Testing, 15(2), 217-263.

Popham, W. J. (2002). Classroom assessment: what teachers need to know (3rd ed.). Boston: Allyn and Bacon.

Reynolds, C. R., Livingston, R. B., \& Willson, V. L. (2006). Measurement and assessment in education. Boston: Pearson/Allyn \& Bacon.

Singer, M,. (2006). A cognitive model for developing a competence based curriculum in secondary education. Current and future challenges in Curriculum: Policies, Practices and Netwroking for change. Bucureşti: Education 2000+ Publishers. Humanitas Educational, pp. 121-141, ISBN-(13) 978973-689-104-5.

\title{
THE CONSTRUCTION OF A LANGUAGE ASSESSMENT COMPETENCE FRAMEWORK FOR PRE-SERVICE ENGLISH LANGUAGE TEACHERS AT ULIS-VNU: A FOCUS ON VALIDITY
}

\author{
Duong Thu Mai, Nguyen Thi Chi, Pham Thi Thu Ha
}

\section{Faculty of English Language Teacher Education, VNU University of Languages and International Studies, Pham Van Dong, Cau Giay, Hanoi, Vietnam}

\begin{abstract}
Given that assessment competence is one of the key professional competence requirements for teachers in general, and English language teachers in particular, this article will address issues concerning constructing such a competence framework, including methodological, theoretical and practical ones. Firstly, the validity of a competence framework will be examined as the fundamental principle of the procedure for framework construction. Subsequently, other theoretical issues and practical concerns in relation to the development of an assessment competence framework for pre-service teachers of English language at Hanoi Vietnam National University will be briefly reviewed before the assessment competence framework is developed and validated by experts. It is in this final step of expert interviewing that the evidence for different validity aspects are considered together in a dynamic relation.
\end{abstract}

Keywords: assessment competence, validity, assessment framework construction 